\title{
Does invasive Chondrostoma nasus shift the parasite community structure of endemic Parachondrostoma toxostoma in sympatric zones?
}

Andrea Šimková1*, Petra Navrátilová1, Martina Dávidová', Markéta Ondračková1, Melthide Sinama², Rémi Chappaz², André Gilles ${ }^{2}$ and Caroline Costedoat ${ }^{2}$

\begin{abstract}
Background: The composition of parasite communities in two cyprinid species in southern France - native and threatened Parachondrostoma toxostoma and introduced Chondrostoma nasus - was investigated. In sympatry, these two species form two hybrid zones in the Durance and Ardeche Rivers. Due to their different feeding preference and habitat positions in allopatry, we supposed a difference in parasite communities between fish species. We expected more similar parasite communities in sympatric zones associated with habitat overlap (facilitating the transmission of ectoparasites) and similar feeding (more generalist behaviour when compared to allopatry, facilitating the transmission of endoparasites) in both fish species. Finally, we investigated whether $P$. toxostoma $\times$ C. nasus hybrids are less parasitized then parental species.
\end{abstract}

Methods: One allopatric population of each fish species plus two sympatric zones were sampled. Fish were identified using cytochrome $b$ gene and 41 microsatellites loci and examined for all metazoan parasites.

Results: A high Monogenea abundance was found in both allopatric and sympatric populations of $C$. nasus. Trematoda was the dominant group in parasite communities of $P$. toxostoma from the allopatric population. In contrast, the populations of $P$. toxostoma in sympatric zones were parasitized by Dactylogyrus species found in $C$. nasus populations, but their abundance in endemic species was low. Consequently, the similarity based on parasite presence/absence between the sympatric populations of $P$. toxostoma and C. nasus was high. Sympatric populations of $P$. toxostoma were more similar than allopatric and sympatric populations of this species. No difference in ectoparasite infection was found between $P$. toxostoma and hybrids, whilst $C$. nasus was more parasitized by Monogenea.

Conclusions: The differences in endoparasites between P. toxostoma and C. nasus in allopatry are probably linked to different feeding or habitat conditions, but host-parasite evolutionary associations also play an important role in determining the presence of Chondrostoma-specific monogeneans. Our findings suggest that Dactylogyrus expanded with the source host $C$. nasus into introduced areas and that $P$. toxostoma became infected after contact with $C$. nasus. Although the genotype of $P$. toxostoma and recombinant genotypes of hybrids are susceptible to Dactylogyrus transmitted from C. nasus, the intensity of infection is low in these genotypes.

Keywords: Biological invasion, Endemic species, Cyprinid fish, Parasite communities, Monogenea, Hybrid zone

\footnotetext{
* Correspondence: simkova@sci.muni.cz

'Department of Botany and Zoology, Faculty of Science, Masaryk University,

Kotlářská 2, 61137 Brno, Czech Republic

Full list of author information is available at the end of the article
} 


\section{Background}

Biologists have long recognized that introduced species may have major effects on native communities. If an invading species occupies the same niche as a native species, strong interactions are likely to occur [1]. The introduction of species into novel areas and the formation of sympatric zones may affect the parasite diversity and distribution in native species. Introduced species may act as a competent host for a native parasite in which the infection is multiplied; then, the parasite "spills back" into the native host. This could result in an increase in the abundance of the native parasite and consequently in an increasing disease impact on native species [2]. By contrast, if an introduced species is resistant to a native parasite, it creates a dilution effect, lowering the parasite infection in the native host $[3,4]$. In addition, although the release from parasites and pathogens is considered to be a key factor explaining the successful expansion and survival of non-native species in the introduced regions (e.g. [5-7]), non-native parasite species may be introduced with their host, potentially threatening endangered and endemic local species [8]. Novel parasites introduced by the invader may remain host specific, or may be transmitted to native species. Especially in related host species, introduced parasites may represent a danger to native species, as they are expected to be adapted to the invader (e.g. [9]). In general, a change in patterns of parasitism may in turn affect host population dynamics.

After introduction events, the contact of native species with non-native relative species in sympatric areas often leads to the interspecific hybridization and formation of a hybrid zone. Hybridization becomes problematic especially for rare and endemic species [10]. Concerning the role of parasitism in hybrid zones, there have been a number of experimental and field studies investigating the resistance of different parental taxa and their hybrids to pathogens or parasites. Some of them using fish, amphibians or mammals as models have suggested that hybrids are less parasitized or more resistant to pathogen infection than their parental species [11-13]. However, other studies have indicated decreasing resistance as a result of hybridization processes [14-17]. It was proposed that hybrid susceptibility resulted from genomic incompatibilities between parental taxa [16]. Derothe et al. $[17,18]$ postulated and experimentally confirmed the "parasite constraint" hypothesis, i.e. hybrid susceptibility is only applied to parasite species that have exerted enough constraints on their host to induce the selection of co-adapted genes among immune genes in the two parental genomes.

Many closely related cyprinid species living in sympatry tend to hybridize (e.g. [19-21]) and some of them form hybrid zones [22,23]. In cyprinids, several previous cases involving introduction of new species have led to the endangerment of native species. The situation with two particular cyprinid species - the native Parachondrostoma toxostoma and the introduced Chondrostoma nasus living in sympatry and forming two hybrid zones in the Durance River (including the Durance river plus the Buech river, which is a tributary) and the Ardeche River (South France, Rhone River drainage) - is such an example. Parachondrostoma toxostoma is a threatened, protected endemic cyprinid species in southern France. Chondrostoma nasus was introduced from Eastern Europe and colonized a part of the distribution range of $P$. toxostoma. The Durance hybrid zone of P. toxostoma and $C$. nasus has, until now, been studied more than the Ardeche hybrid zone. The Durance hybrid zone is of recent origin (around 100-150 years old) and represents a complex system with multiple effects including interspecies competition, bidirectional introgression, and environmental pressures [24]. The absence of a reproductive barrier between these two species found by Costedoat et al. [23] facilitates the hybridization between $P$. toxostoma and C. nasus.

Both species are morphologically and ecologically well differentiated in allopatry. Because of the different morphology of the mouth [25], P. toxostoma has a more generalist diet, feeding on algae and invertebrates, the latter being intermediate hosts for endoparasites, while C. nasus is a more specialized feeder, feeding mainly on benthic diatoms and algae in allopatric populations [26-29]. A difference in mouth morphology between allopatric and sympatric specimens and a convergence in mouth shape in the Durance hybrid zone were observed [30].

The aim of the study was to analyze the metazoan parasite communities of $P$. toxostoma and C. nasus. Due to different feeding preferences in allopatry, we supposed that allopatric $P$. toxostoma would be more parasitized by endoparasites than allopatric C. nasus. We also supposed differences in the composition of parasite communities between allopatric and sympatric populations. However, we expected the existence of more similar parasite fauna between $P$. toxostoma and $C$. nasus in sympatric areas where contact between the two species facilitates the transmission of ectoparasites, and we further expected that a shift to a larger generalist diet should facilitate the transmission of endoparasites. Finally, we investigated the parasite fauna of $P$. toxostoma $\mathrm{x} C$. nasus hybrids and compared them with the parasite fauna of pure species.

\section{Methods}

Four field studies (end of August 2008, June 2010, September 2010, and June 2011) were performed to collect Parachondrostoma/Chondrostoma specimens in southern France. One allopatric population of each spe- 
cies was sampled, Parachondrostoma toxostoma from the Orbieu River (Mediterranean Coastal river) and C. nasus from the Allier River (Loire drainage). Four localities on the Buech-Durance Rivers (Avignon, Manosque, Pertuis, Pont de Laragne) and two localities on the Ardeche River (Saint Just and Labeaume), where both fish species live in sympatry (two sympatric zones correspond to two hybrid zones in our study), were sampled (Figure 1, Table 1). The periods of investigation were selected to eliminate the effect of temporal variability (i.e. seasonality) on the composition of parasite communities and to exclude hot summers when fish collection and transport to the laboratory is difficult to perform. Fish were caught using electrofishing and transported live to the laboratory in barrels with the original oxygenated water. All fish were killed by severing the spinal cord shortly before dissection. The standard length (in millimeters) of each specimen was recorded. Complete dissection of fish was performed following Ergens \& Lom [31]. Fish were examined for all metazoan parasites - ectoparasites (Monogenea, Crustacea, Mollusca and Hirudinea) and endoparasites (Trematoda, Cestoda, Acanthocephala and Nematoda). All parasites were removed, fixed as described in Rohlenová et al. [32], and determined to species/genus level using a light microscope (Olympus BX50) equipped with phase-contrast, differential interference contrast (DIC) and Digital Image Analysis (Olympus MicroImage ${ }^{\text {TM }}$ for Windows 95/98/ NT 4.0 (Olympus Optical Co.)).

\section{Molecular identification}

A fin sample from each fish specimen was preserved in $70 \%$ ethanol. All specimens were identified genetically using mitochondrial ( 5 ' part of the cytochrome $b$ gene, as described in Costedoat et al. [33]) and nuclear markers (41 microsatellites distributed in five multiplex PCR kits, [34]). For microsatellites, amplification was performed for each kit in a total volume of $10 \mu \mathrm{L}$ containing $1 \mu \mathrm{L}$ of total DNA extract using Qiagen Multiplex PCR Kit following the manufacturer's protocol. Thermocycling was performed on a Mastercycler gradient (Eppendorf) with the following protocol: $95^{\circ} \mathrm{C}$ for $10 \mathrm{~min}$, followed by 30 cycles $\left(94^{\circ} \mathrm{C}\right.$ for $1 \mathrm{~min}, 57^{\circ} \mathrm{C}$ for $1 \mathrm{~min}, 72^{\circ} \mathrm{C}$ for $1 \mathrm{~min}$ ), and $60^{\circ} \mathrm{C}$ for $45 \mathrm{~min}$. Visualization of the amplicons was conducted in an ABI 3130 Genetic Analyzer (Applied Biosystems). Allele sizes were scored against an internal GeneScan500 LIZ standard and genotypes were obtained using GeneMapper $^{\circledR} 3.7$ [35].

\section{Data analyses}

MicroChecker 2.2.3 [36] was used to check for null alleles, or scoring errors resulting from stuttering. NewHybrid 1.1 [37], a Bayesian clustering method, was used to assign specimens to genotypic classes: pure species ( $C$. nasus and P. toxostoma) in allopatric and sympatric zones plus hybrids in sympatric zones. The Markov Chain Monte Carlo (MCMC) model was applied to compute all

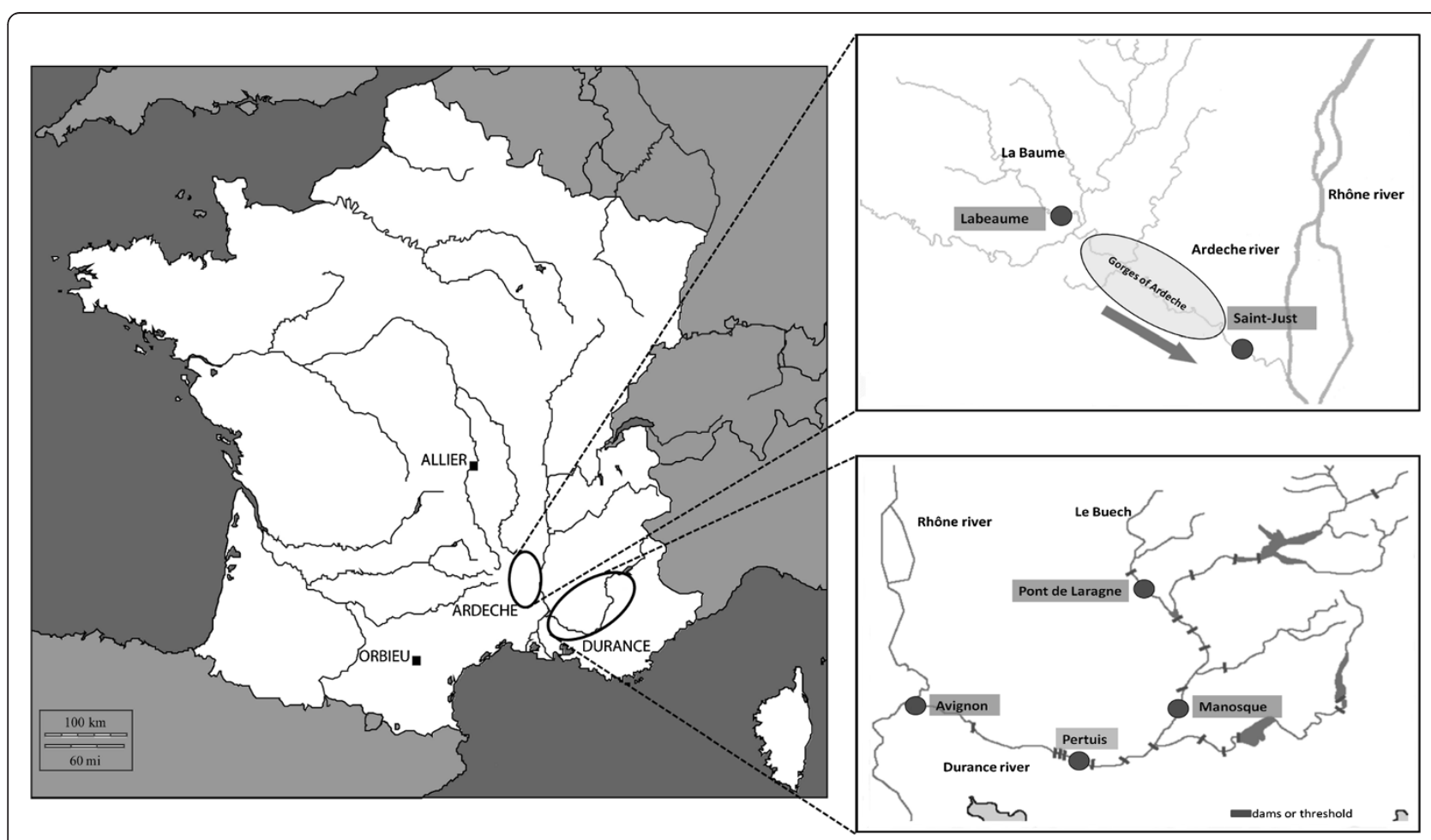

Figure 1 Positions of the localities investigated in southern France. 
Table 1 Locality description and molecular identification of Parachondrostoma toxostoma (PT), Chondrostoma nasus (CN), and hybrid (H) specimens

\begin{tabular}{llll}
\hline \multicolumn{1}{c}{ Localities } & Type of population & $\mathbf{N}$ & \multicolumn{1}{c}{ Identification } \\
\hline Allier & allopatric CN & 11 & $11 \mathrm{CN}$ \\
Orbieu & allopatric PT & $1111 \mathrm{PT}$ \\
Le Buech (Pont de Laragne) & sympatric & $4117 \mathrm{PT}, 19 \mathrm{CN}, 5 \mathrm{H}$ \\
Durance (Avignon) & sympatric & $3633 \mathrm{CN}, 3 \mathrm{H}$ \\
Durance (Manosque) & sympatric & $6849 \mathrm{PT}, 9 \mathrm{CN}, 10 \mathrm{H}$ \\
Durance (Pertuis) & sympatric & $2220 \mathrm{PT}, 1 \mathrm{CN}, 1 \mathrm{H}$ \\
Ardeche (Saint Just) & sympatric & $3011 \mathrm{PT}, 18 \mathrm{CN}, 1 \mathrm{H}$ \\
La Baume (Labeaume) & sympatric & $2419 \mathrm{PT}, 5 \mathrm{H}$ \\
\hline
\end{tabular}

$\mathrm{N}$ represents the number of fish identified with molecular markers.

probabilities that one individual belongs to these different classes. The program was run five times with varying lengths of burn-in period and numbers of sweeps.

Adze 1.0 [38] was applied to estimate allelic richness and private allelic richness using the rarefaction method to compare populations with different sample sizes. The allelic richness of a population is the expected number of alleles in a sample of genes taken from a population. The private allelic richness is a convenient measure of how distinct a population is from other populations. Arlequin 3.1 [39] was used to estimate observed and expected heterozygosity by population. Genhet 2.3 [40] function implemented in $\mathrm{R}$ software ( $\mathrm{R}$ development core team 2010) was used to calculate the standardized heterozygosity based on the mean expected heterozygosity and the standardized heterozygosity based on the mean observed heterozygosity. ANOVA with the Tukey post hoc test was used to test differences in heterozygosity between $P$. toxostoma, C. nasus and hybrids.

Similarity in parasite communities was calculated using the qualitative Jaccard index on the presence/ absence matrix and the quantitative Morisita index on parasite abundance data [41]. General linear models (GLM) using the boostrap test with 1,000 permutations were applied to test differences in similarity among the following groups of populations (C. nasus - C. nasus; $P$. toxostoma - P. toxostoma; $C$. nasus $-P$. toxostoma) using (1) all populations and (2) only sympatric populations. Sample size and fish body length were included in GLM as covariates. Post hoc tests with Bonferroni correction were applied for multiple comparisons. In addition, GLM were applied to test the differences in similarity between "allopatric-sympatric" and "sympatricsympatric" groups when the "sympatric-sympatric" group included the pairs of populations from both zones where $C$. nasus and $P$. toxostoma live in sympatry. This comparison was made for each fish species. The effect of host - i.e. P. toxostoma, C. nasus or hybrids - on parasite species richness or parasite abundance was tested using ANCOVA with fish body length and microsatellite heterozygosity as covariates. Parasite abundance and parasite species richness were log-transformed prior to GLM and ANCOVA. Statistical analyses were performed in Statistica 10 for Windows, StatSoft Inc and SPSS 20.0.0 (IBM Corporation, 2011).

\section{Results \\ Molecular profiles of Chondrostoma/Parachondrostoma in localities}

Using molecular determination (Table 1), an allopatric population of C. nasus was confirmed at Allier and an allopatric population of $P$. toxostoma was confirmed at Orbieu. Considering the localities where P. toxostoma and C. nasus live in sympatry, three different profiles based on molecular data were found: (1) localities with a high occurrence of $P$. toxostoma (i.e. Labeaume, Pertuis and Manosque), (2) localities with similar proportions of $P$. toxostoma and $C$. nasus (i.e. Pont de Laragne and Saint Just) and (3) a locality with a high occurrence of $C$. nasus (i.e. Avignon). The proportion of hybrids in localities with a sympatric occurrence of $C$. nasus and P. toxostoma was variable (i.e. from 3 to 20\%). The highest proportion of hybrids was found at Manosque (Durance River) and Labeaume (Ardeche River), 16 and 20\% respectively.

\section{Parasitism in sympatric and allopatric Parachondrostoma/ Chondrostoma populations}

A total of 11 fish populations were analyzed, two of which were considered to be allopatric and nine, sympatric (see Table 2 for the populations). Among parasite groups, Monogenea reached high proportions in the allopatric population of $C$. nasus and in all populations of both $P$. toxostoma and $C$. nasus in sympatric zones (Figure 2). However, a decrease in the proportion of Monogenea and an increase in the proportions of Crustacea and/or Trematoda were found in all P. toxostoma populations compared to C. nasus populations in sympatric zones. By contrast, Trematoda achieved the highest proportion in the allopatric population of P. toxostoma. The proportions of other parasite groups were low (Figure 2).

There was a trend towards higher allelic richness and heterozygosity and lower private allelic richness in $C$. nasus populations compared to P. toxostoma. However, such a trend was not found for parasite diversity (Table 2). Dactylogyrus achieved the highest parasite abundance and prevalence in the allopatric population of $C$. nasus and all populations of $C$. nasus in sympatric zones (Table 3), but its abundance in the populations of $P$. toxostoma in sympatric zones was low (four populations) or reached a moderate value (Labeaume) (Table 4). Dactylogyrus ergensi was the most abundant species in parasite component communities of $C$. nasus from the 
Table 2 Microsatellite diversity and parasite diversity (Brillouin index) for P. toxostoma (PT) and C. nasus (CN) populations

\begin{tabular}{|c|c|c|c|c|c|c|c|c|}
\hline Localities & Population & Sample size & Standard length & Allelic richness & $\begin{array}{l}\text { Private allelic } \\
\text { richness }\end{array}$ & Ho & $\mathrm{He}$ & $\begin{array}{l}\text { Brillouin index of } \\
\text { parasite diversity }\end{array}$ \\
\hline Orbieu & PT & 11 & $176 \pm 10.14$ & $2.03 \pm 0.17$ & $0.15 \pm 0.05$ & $0.47 \pm 0.27$ & $0.49 \pm 0.22$ & $1.02 \pm 0.22$ \\
\hline Allier & $\mathrm{CN}$ & 11 & $189.55 \pm 28.44$ & $3.03 \pm 0.20$ & $0.14 \pm 0.04$ & $0.61 \pm 0.18$ & $0.64 \pm 0.17$ & $0.87 \pm 0.24$ \\
\hline \multirow[t]{2}{*}{ Pertuis } & PT & 20 & $128.65 \pm 11.61$ & $2.97 \pm 0.30$ & $0.26 \pm 0.06$ & $0.51 \pm 0.31$ & $0.53 \pm 0.30$ & $0.56 \pm 0.39$ \\
\hline & $\mathrm{CN}$ & 1 & 100 & - & - & - & - & - \\
\hline \multirow[t]{2}{*}{ Manosque } & PT & 49 & $113.61 \pm 22.66$ & $2.95 \pm 0.29$ & $0.26 \pm 0.06$ & $0.48 \pm 0.30$ & $0.50 \pm 0.30$ & $0.37 \pm 0.36$ \\
\hline & $\mathrm{CN}$ & 9 & $130 \pm 6.80$ & $3.46 \pm 0.23$ & $0.23 \pm 0.04$ & $0.60 \pm 0.26$ & $0.66 \pm 0.22$ & $0.75 \pm 0.46$ \\
\hline \multirow[t]{2}{*}{ Avignon } & PT & - & - & - & - & - & - & - \\
\hline & $\mathrm{CN}$ & 33 & $203.41 \pm 37.01$ & $3.39 \pm 0.23$ & $0.16 \pm 0.04$ & $0.61 \pm 0.21$ & $0.65 \pm 0.20$ & $0.63 \pm 0.37$ \\
\hline \multirow[t]{2}{*}{ Pont de Laragne } & PT & 17 & $117.24 \pm 15.41$ & $2.84 \pm 0.29$ & $0.23 \pm 0.06$ & $0.54 \pm 0.30$ & $0.55 \pm 0.28$ & $0.44 \pm 0.41$ \\
\hline & $\mathrm{CN}$ & 19 & $155.47 \pm 43.75$ & $3.27 \pm 0.22$ & $0.15 \pm 0.03$ & $0.63 \pm 0.22$ & $0.64 \pm 0.19$ & $0.42 \pm 0.39$ \\
\hline \multirow[t]{2}{*}{ LaBeaume } & PT & 19 & $175.89 \pm 18.77$ & $3.03 \pm 0.29$ & $0.25 \pm 0.06$ & $0.54 \pm 0.30$ & $0.52 \pm 0.31$ & $1.31 \pm 0.23$ \\
\hline & $\mathrm{CN}$ & - & & - & - & - & - & \\
\hline \multirow[t]{2}{*}{ Saint Just } & PT & 11 & $108.18 \pm 10.31$ & $2.93 \pm 0.30$ & $0.27 \pm 0.09$ & $0.53 \pm 0.31$ & $0.55 \pm 0.29$ & $0.61 \pm 0.47$ \\
\hline & CN & 18 & $113.22 \pm 25.96$ & $3.43 \pm 0.23$ & $0.19 \pm 0.04$ & $0.64 \pm 0.24$ & $0.65 \pm 0.20$ & $1.04 \pm 0.37$ \\
\hline
\end{tabular}

Ho - observed heterozygosity, He - expected heterozygosity. Mean with standard deviation are shown.

allopatric population and three sympatric populations (Table 3). Dactylogyrus dirigerus and Dactylogyrus new sp. (this species is morphologically closely related to $D$. ergensi and $D$. dirigerus) were dominant in the parasite component community of $C$. nasus from Pont de Laragne (Table 3). Dactylogyrus chondrostomi was found in four populations of $C$. nasus, whilst this parasite species only rarely occurred in the $P$. toxostoma population at Manosque. In the allopatric population of P. toxostoma, only Dactylogyrus vistulae was found; this species achieved low abundance and prevalence (Table 4). Among Crustacea, Ergasilus sieboldi achieved high abundance and prevalence in two $P$. toxostoma populations (Table 4). Concerning endoparasites, Diplostomum spp. was the most abundant and prevalent in all populations of $P$. toxostoma and C. nasus in sympatric zones.

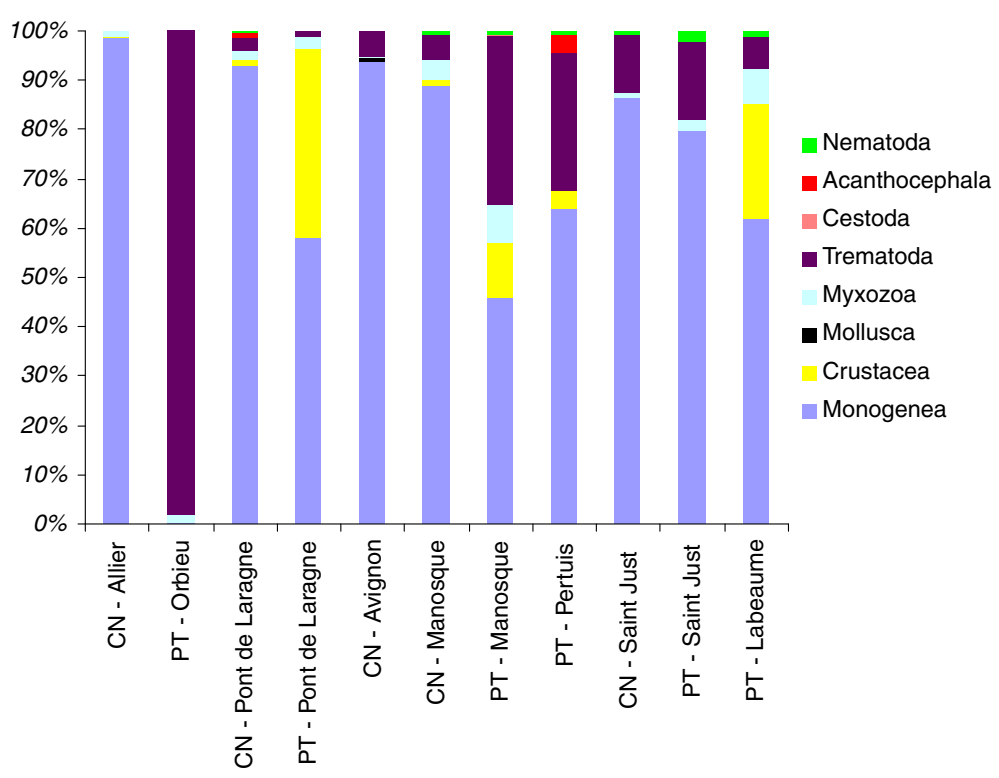

Figure 2 Short title: Metazoan parasites in C. nasus and P. toxostoma populations. Detail legend: Proportions of metazoan parasite groups in C. nasus and P. toxostoma populations. For sample size of each population, see Table 2. 
Table 3 Parasite abundance ( $A$, mean \pm standard deviation) and prevalence $(P$, in $\%)$ for each parasite species in $C$. nasus populations

\begin{tabular}{|c|c|c|c|c|c|c|c|c|c|c|c|c|c|c|}
\hline \multirow[t]{2}{*}{ Parasite species } & \multicolumn{2}{|l|}{ Allier (11) } & \multicolumn{2}{|c|}{ Pont de Laragne (19) } & \multirow{2}{*}{$H(5)$} & \multicolumn{2}{|c|}{ Avignon (33) } & \multirow[b]{2}{*}{$\mathrm{H}(3)$} & \multicolumn{2}{|c|}{ Manosque (9) } & \multirow{2}{*}{$H(10)$} & \multicolumn{2}{|c|}{ Saint Just (18) } & \multirow{2}{*}{$H(1)$} \\
\hline & A & $P$ & A & $\mathbf{P}$ & & $A$ & $P$ & & A & $P$ & & A & $\mathbf{P}$ & \\
\hline Dactylogyrus chondrostomi & $2.27 \pm 2.63$ & 55 & - & - & & $3.15 \pm 5.29$ & 70 & * & $1.22 \pm 1.79$ & 44 & & $2.33 \pm 4.47$ & 56 & \\
\hline Dactylogyrus dirigerus & $6.82 \pm 9.61$ & 73 & $7.42 \pm 17.62$ & 58 & * & $2 \pm 2.41$ & 64 & * & $1.11 \pm 1.54$ & 44 & * & $1.89 \pm 2.47$ & 56 & * \\
\hline Dactylogyrus ergensi & $65.64 \pm 77.79$ & 100 & - & - & * & $44.91 \pm 65.77$ & 91 & * & $15.89 \pm 12.62$ & 89 & $*$ & $9.5 \pm 13.5$ & 72 & \\
\hline Dactylogyrus new sp. & $4.18 \pm 6.01$ & 64 & $19.16 \pm 65.06$ & 47 & * & $1.45 \pm 2.43$ & 52 & * & $3.33 \pm 5.05$ & 56 & * & $2.72 \pm 2.52$ & 67 & * \\
\hline Dactylogyrus vistulae & $14.18 \pm 15.33$ & 100 & $0.26 \pm 0.56$ & 21 & * & $1.21 \pm 1.62$ & 52 & * & $0.11 \pm 0.33$ & 11 & * & $4.11 \pm 3.94$ & 89 & * \\
\hline Gyrodactylus macrocornis & - & - & $0.26 \pm 0.73$ & 16 & * & $0.24 \pm 0.56$ & 18 & & $2.78 \pm 3.27$ & 67 & $*$ & $0.28 \pm 0.75$ & 17 & \\
\hline Gyrodactylus pannonicus & - & - & $0.37 \pm 1.12$ & 11 & * & - & - & & - & - & * & - & - & \\
\hline Gyrodactylus paraminimus & $0.09 \pm 0.29$ & 9 & - & - & & $0.09 \pm 0.38$ & 6 & & $0.33 \pm 0.71$ & 22 & & $0.61 \pm 1.34$ & 22 & \\
\hline Paradiplozoon homoion & - & - & - & - & & - & - & & - & - & & $0.06 \pm 0.24$ & 6 & \\
\hline Paradiplozoon new sp. & $0.09 \pm 0.29$ & 9 & - & - & & $0.09 \pm 0.29$ & 9 & * & - & - & & $0.06 \pm 0.24$ & 6 & \\
\hline Argulus foliaceus & - & - & - & - & & - & - & & - & - & $*$ & $0.06 \pm 0.24$ & 6 & \\
\hline Ergasilus sieboldi & - & - & $0.16 \pm 0.50$ & 11 & * & - & - & & $0.33 \pm 1$ & 11 & * & - & - & \\
\hline Lamproglena pulchella & $0.18 \pm 0.39$ & 18 & $0.16 \pm 0.50$ & 11 & & - & - & & - & - & & - & - & \\
\hline Anodonta sp. & - & - & - & - & & $0.36 \pm 1.19$ & 12 & * & - & - & & - & - & \\
\hline Myxobolus spp. & $1.18 \pm 2.21$ & 27 & $0.63 \pm 1.74$ & 16 & * & $0.18 \pm 0.73$ & 9 & & $1.11 \pm 2.62$ & 33 & * & $0.28 \pm 0.46$ & 28 & \\
\hline Allocreadium markewitschi & - & - & - & - & & - & - & & - & - & & $0.33 \pm 1.41$ & 6 & \\
\hline Apharyngostrigea cornu & - & - & - & - & & - & - & & - & - & & $0.06 \pm 0.24$ & 6 & \\
\hline Clinostomum complanatum & - & - & - & - & & - & - & & - & - & & - & - & \\
\hline Diplostomum spp. & - & - & $0.74 \pm 1.56$ & 21 & & $2.97 \pm 6.87$ & 39 & * & $1.44 \pm 3.28$ & 33 & & $2.39 \pm 3.68$ & 56 & * \\
\hline Echinostomatidae fam. sp. & - & - & - & - & & - & - & & - & - & & $0.06 \pm 0.24$ & 6 & \\
\hline Metorchis xanthosomus & - & - & - & - & & - & - & & - & - & & - & - & \\
\hline Paryphostomum radiatum & - & - & - & - & & - & - & * & - & - & & $0.11 \pm 0.32$ & 11 & \\
\hline Trematoda sp. metac. & - & - & - & - & & - & - & & - & - & & - & - & \\
\hline Neogryporhynchus chleilancristrotus & - & - & - & - & & - & - & & - & - & & - & - & \\
\hline Pomphorhynchus terreticolis & - & - & $0.32 \pm 0.82$ & 16 & & - & - & * & - & - & & - & - & \\
\hline Contracoecum sp. & - & - & - & - & & - & - & & $0.11 \pm 0.33$ & 11 & & - & - & \\
\hline Cucullanus sp. & - & - & - & - & & - & - & & - & - & & - & - & \\
\hline Philometra sp. & - & - & $0.05 \pm 0.23$ & 5 & & - & - & & $0.11 \pm 0.33$ & 11 & & - & - & \\
\hline Pseudocapillaria sp. & - & - & - & - & & - & - & & - & - & & - & - & \\
\hline Raphidascaris acus & - & - & $0.05 \pm 0.23$ & 5 & & - & - & & - & - & & $0.17 \pm 0.71$ & 6 & \\
\hline
\end{tabular}

The presence of parasite species in hybrids $(\mathrm{H})$ is indicated by asterisks; sample size is included parentheses.

The abundance of other endoparasite species in $P$. toxostoma and $C$. nasus populations in sympatric zones was low. In the allopatric population of P. toxostoma, four species of Trematoda were highly prevalent and abundant (Table 4).

\section{Similarity in parasite communities in Parachondrostoma/ Chondrostoma populations}

The similarity of parasite communities based on parasite presence/absence data or parasite abundance for different pairs of populations is shown in Figure 3A-B. The lowest similarity in parasite communities was found between the allopatric population of $C$. nasus and the allopatric population of $P$. toxostoma using both parasite abundance and parasite presence data. When considering all populations, a significant difference in the similarity based on parasite abundance was found among the pairs of $C$. nasus - C. nasus; P. toxostoma - P. toxostoma; and P. toxostoma - C. nasus populations (whole model $\mathrm{F}_{4,50}=3.60, \mathrm{p}=0.012, \quad$ similarity $\left.\mathrm{F}=6.69, \mathrm{p}=0.003\right)$. Using the post hoc test, a significantly higher similarity was found only between $C$. nasus - C. nasus populations than between $P$. toxostoma $-C$. nasus populations ( $\mathrm{p}=0.003$ after Bonferroni correction). No significant difference in similarity among the pairs of populations based on parasite presence data was found ( $p>0.05)$. 
Table 4 Parasite abundance $(A$, mean \pm standard deviation) and prevalence $(P$, in $\%)$ for each parasite species in $P$. toxostoma populations

\begin{tabular}{|c|c|c|c|c|c|c|c|c|c|c|c|c|c|c|c|c|c|}
\hline \multirow[t]{2}{*}{ Parasite species } & \multicolumn{2}{|c|}{ Orbieu (11) } & \multicolumn{2}{|c|}{$\begin{array}{l}\text { Pont de Laragne } \\
\text { (17) }\end{array}$} & \multirow{2}{*}{$H(5)$} & \multicolumn{2}{|c|}{$\begin{array}{l}\text { Manosque } \\
\text { (49) }\end{array}$} & \multirow{2}{*}{$H(10)$} & \multicolumn{2}{|l|}{$\begin{array}{l}\text { Pertuis } \\
\text { (20) }\end{array}$} & \multirow[b]{2}{*}{$H(1)$} & \multicolumn{2}{|c|}{$\begin{array}{l}\text { Saint Just } \\
\text { (11) }\end{array}$} & & \multicolumn{2}{|c|}{$\begin{array}{l}\text { Labeaume } \\
\text { (19) }\end{array}$} & \multirow[b]{2}{*}{$\mathrm{H}(5$} \\
\hline & A & $\mathbf{P}$ & A & $P$ & & A & $\bar{P}$ & & A & $\bar{P}$ & & A & & & A & $\bar{P}$ & \\
\hline Dactylogyrus chondrostomi & - & - & - & - & & $0.04 \pm 0.2$ & 4 & & - & - & & - & - & & - & - & \\
\hline Dactylogyrus dirigerus & - & - & $0.18 \pm 0.39$ & 18 & * & $0.10 \pm 0.47$ & 6 & * & $0.4 \pm 1.19$ & 15 & & $1.27 \pm 1.74$ & 55 & $*$ & $5.79 \pm 5.41$ & 84 & * \\
\hline Dactylogyrus ergensi & - & - & $0.65 \pm 0.93$ & 41 & * & $1 \pm 1.79$ & 39 & * & $0.45 \pm 0.69$ & 35 & & $0.09 \pm 0.3$ & 9 & & $4.63 \pm 5.45$ & 84 & * \\
\hline Dactylogyrus new sp. & - & - & $0.35 \pm 0.86$ & 18 & * & $0.35 \pm 0.81$ & 22 & * & $0.25 \pm 0.72$ & 15 & & $1.54 \pm 1.44$ & 64 & * & $6.11 \pm 7.67$ & 89 & * \\
\hline Dactylogyrus vistulae & $0.09 \pm 0.29$ & 9 & $0.24 \pm 0.56$ & 18 & * & $0.29 \pm 0.58$ & 22 & * & $2.25 \pm 2.69$ & 75 & & $2.27 \pm 4$ & 45 & * & $7 \pm 5.65$ & 89 & * \\
\hline Gyrodactylus macrocornis & $0.09 \pm 0.29$ & 9 & $1.82 \pm 6.26$ & 29 & * & $0.37 \pm 0.97$ & 16 & * & $0.25 \pm 0.72$ & 15 & $*$ & $0.27 \pm 0.65$ & 18 & & $0.74 \pm 1.33$ & 32 & * \\
\hline Gyrodactylus pannonicus & - & - & $5.71 \pm 17$ & 47 & * & $0.61 \pm 2.75$ & 16 & * & $0.3 \pm 0.92$ & 15 & & - & - & & - & - & \\
\hline Gyrodactylus paraminimus & - & - & $0.06 \pm 0.24$ & 6 & & $0.12 \pm 0.33$ & 12 & & - & - & & - & - & & $0.05 \pm 0.23$ & 5 & \\
\hline Paradiplozoon homoion & - & - & - & - & & - & - & & $0.45 \pm 0.76$ & 30 & & $0.45 \pm 0.82$ & 27 & & - & - & \\
\hline Paradiplozoon new sp. & - & - & - & - & & - & - & & - & - & & $0.55 \pm 1.04$ & 27 & & - & - & \\
\hline Argulus foliaceus & - & - & - & - & & - & - & * & - & - & & - & - & & $0.32 \pm 0.58$ & 26 & \\
\hline Ergasilus sieboldi & $0.09 \pm 0.29$ & 9 & $5.88 \pm 17.33$ & 41 & * & $0.63 \pm 1.73$ & 24 & * & $0.25 \pm 0.72$ & 15 & & - & - & & $8.95 \pm 16.75$ & 89 & * \\
\hline Lamproglena pulchella & - & - & - & - & & $0.06 \pm 0.24$ & 6 & & - & - & & - & - & & - & - & \\
\hline Anodonta sp. & - & - & - & - & & - & - & & - & - & & - & - & & - & - & \\
\hline Myxobolus spp. & $0.82 \pm 1.27$ & 36 & $0.41 \pm 1.23$ & 18 & $*$ & $0.49 \pm 1.91$ & 10 & * & - & - & & $0.18 \pm 0.6$ & 9 & & $2.68 \pm 4.62$ & 42 & * \\
\hline Allocreadium markewitschi & - & - & - & - & & - & - & & - & - & & - & - & & - & - & \\
\hline Apharyngostrigea cornu & - & - & - & - & & - & - & & - & - & & $0.09 \pm 0.3$ & 9 & & $0.05 \pm 0.23$ & 5 & \\
\hline $\begin{array}{l}\text { Clinostomum } \\
\text { complanatum }\end{array}$ & $0.18 \pm 0.39$ & 18 & - & - & & - & - & & - & - & & - & - & & $0.63 \pm 1.5$ & 26 & \\
\hline Diplostomum spp. & $13.36 \pm 9.6$ & 100 & $0.18 \pm 0.53$ & 12 & & $2.12 \pm 4.33$ & 35 & & $1.8 \pm 2.24$ & 55 & * & $1.18 \pm 2.99$ & 27 & * & $1.95 \pm 2.61$ & 58 & * \\
\hline Echinostomatidae fam. sp. & $0.64 \pm 2.01$ & 9 & - & - & & - & - & & - & - & & - & - & & - & - & \\
\hline Metorchis xanthosomus & $8.36 \pm 9.72$ & 82 & - & - & & $0.02 \pm 0.14$ & 2 & & - & - & & - & - & & - & - & \\
\hline Paryphostomum radiatum & $29.36 \pm 16.91$ & 100 & - & - & & $0.02 \pm 0.14$ & 2 & & - & - & & - & - & & - & - & \\
\hline Trematoda sp. metac. & $11.91 \pm 10.30$ & 91 & - & - & & - & - & & $0.1 \pm 0.45$ & 5 & & - & - & & - & - & \\
\hline $\begin{array}{l}\text { Neogryporhynchus } \\
\text { chleilancristrotus }\end{array}$ & - & - & - & - & & $0.02 \pm 0.14$ & 2 & & - & - & & - & - & & - & - & \\
\hline $\begin{array}{l}\text { Pomphorhynchus } \\
\text { terreticolis }\end{array}$ & - & - & - & - & & - & - & & $0.25 \pm 0.64$ & 15 & & - & - & & - & - & \\
\hline Contracoecum sp. & - & - & - & - & & - & - & & - & - & & - & - & & - & - & \\
\hline Cucullanus sp. & - & - & - & - & & - & - & & $0.05 \pm 0.22$ & 5 & & - & - & & - & - & \\
\hline Philometra sp. & - & - & - & - & & $0.04 \pm 0.2$ & 4 & & - & - & & - & - & & - & - & \\
\hline Pseudocapillaria sp. & - & - & - & - & & - & - & & - & - & & $0.18 \pm 0.6$ & 9 & & $0.47 \pm 1.5$ & 10 & \\
\hline Raphidascaris acus & - & - & - & - & & - & - & & - & - & & - & - & & - & - & \\
\hline
\end{tabular}

When comparing the similarity in parasite communities of $C$. nasus populations, no difference between allopatric-sympatric and sympatric-sympatric pairs of populations was found $(\mathrm{p}>0.05)$. However, when comparing the similarity in parasite communities of P. toxostoma populations, a significantly higher similarity between sympatric-sympatric pairs of populations than between allopatric-sympatric pairs of populations was found using both parasite abundance (whole model $\mathrm{F}_{3,11}=4.71$, $\mathrm{p}=0.024$, similarity $\mathrm{F}=12.26, \mathrm{p}=0.005)$ and parasite presence (whole model $\mathrm{F}_{3,11}=3.68, \mathrm{p}=0.047$, similarity $\mathrm{F}=10.26, \mathrm{p}=0.008$ ). Moreover, the similarity in parasite communities based on parasite presence/absence data between sympatric $P$. toxostoma and $C$. nasus populations was higher than that between allopatric and sympatric populations of $P$. toxostoma (whole model $\left.\mathrm{F}_{3,21}=6.18, \mathrm{p}=0.004, \mathrm{~F}=14.90, \mathrm{p}=0.001\right)$. When considering only the populations from two sympatric zones, there was no significant difference in the similarity of parasite communities among C. nasus - C. nasus; P. toxostoma - 


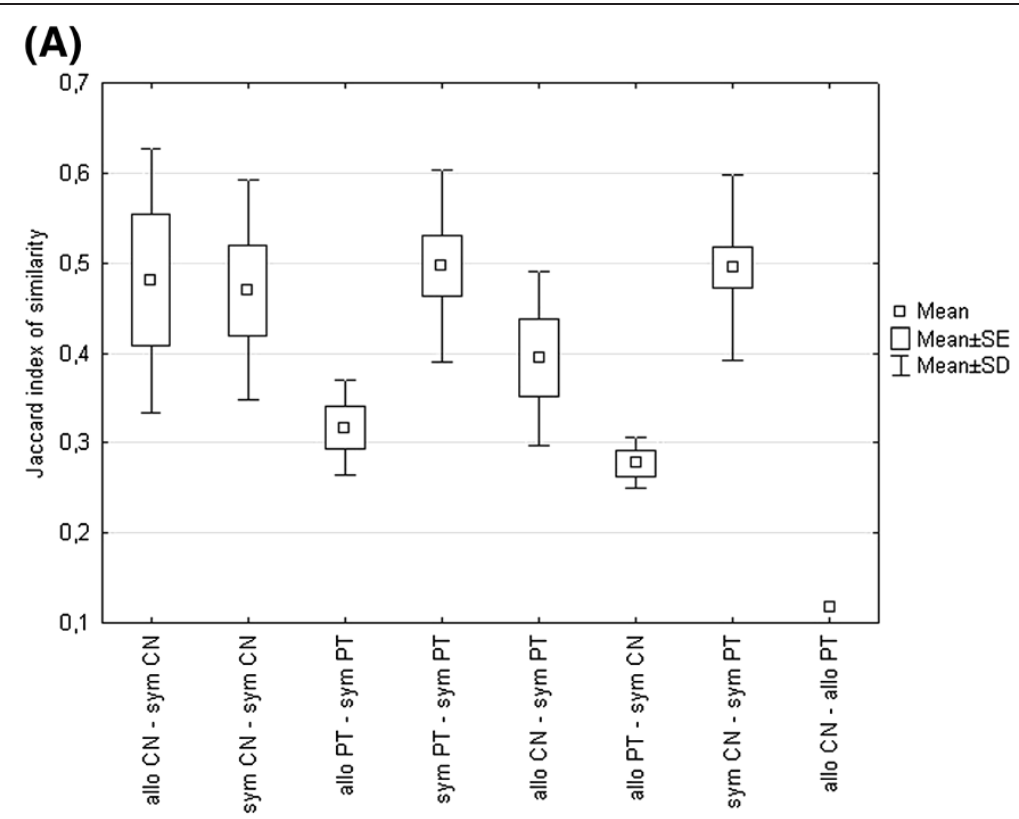

(B)

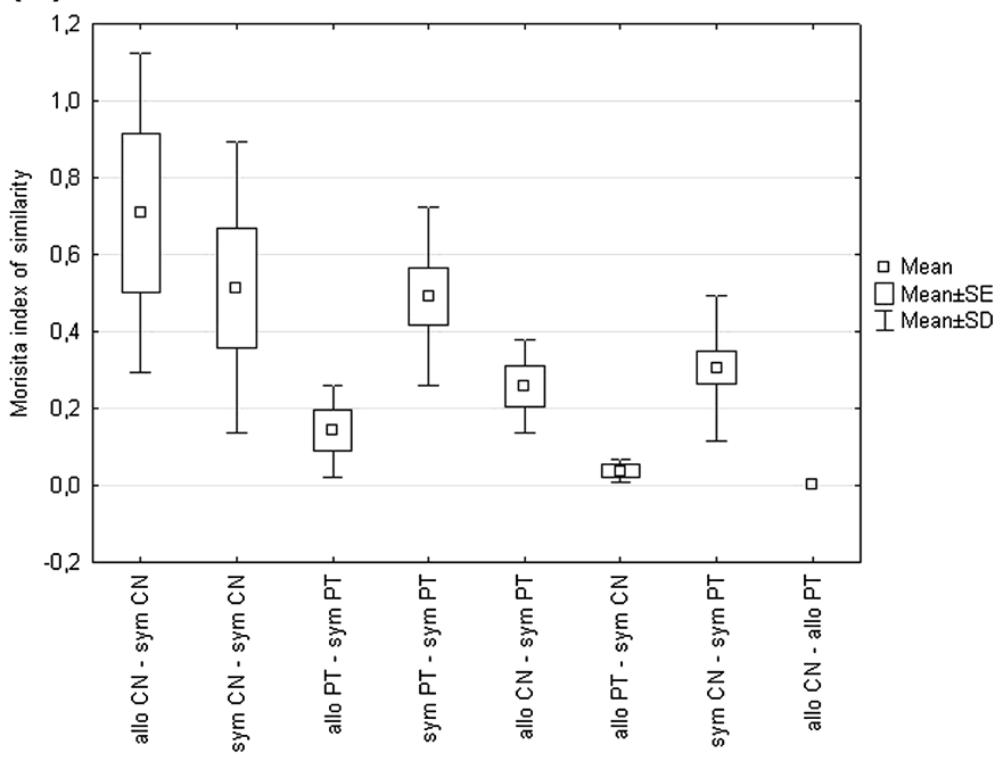

Figure 3 Short title: Similarity in parasite communities based on parasite presence/absence data and parasite abundance. Detail legend: Similarity in parasite communities based on parasite presence/absence data using Jaccard index (A), and parasite abundance using Morisita index (B) between different pairs of populations; allo - allopatric population; sym - sympatric population; CN - Chondrostoma nasus; PT -

Parachondrostoma toxostoma.

P. toxostoma; or P. toxostoma - C. nasus pairs of populations based on parasite abundance or parasite presence data ( $\mathrm{p}>0.05)$. Host sample size and body length were insignificant in all GLM showed below.

\section{Parasitism in P. toxostoma and C. nasus hybrids}

The molecular profiles of Chondrostoma/Parachondrostoma populations in hybrid zones (see Table 1) did not allow us to test simultaneously the effect of locality and effect of host on parasitism. Therefore, first, only the effect of host was tested using the dataset of all localities from sympatric zones. Microsatellite heterozygosity differed between P. toxostoma, C. nasus and hybrids (ANOVA, $\mathrm{F}_{2}$, $220=99.39, \mathrm{p}<0.001)$. The heterozygosity of hybrids was higher than that of both pure species and the heterozygosity of $C$. nasus was higher than that of $P$. toxostoma $(\mathrm{p}<0.001)$. A significant effect of host on ectoparasite abundance was found (ANCOVA, $\mathrm{F}_{4,215}$ total $=42.31$, $\mathrm{p}<0.001$, heterozygosity $\mathrm{F}=1.99, \mathrm{p}=0.159$, body length $\mathrm{F}=116.03, \mathrm{p}<0.001$, host $\mathrm{F}=5.01, \mathrm{p}=0.007)$. The Tukey 
post hoc test revealed significantly higher ectoparasite abundance in $C$. nasus when compared with $P$. toxostoma and hybrids $(\mathrm{p}<0.001)$. The same result was found when using Monogenea or Dactylogyrus abundance. The prevalence of Dactylogyrus chondrostomi (a parasite species specific to $C$. nasus) in hybrids in the whole sample of sympatric zones was low (12\%) and this species was found only in hybrids at Avignon. A significant effect of host on endoparasite abundance was also found (ANCOVA, $\mathrm{F}_{4,215}$ total $=6.56, \mathrm{p}<0.001$, heterozygosity $\mathrm{F}=2.05, \mathrm{p}=0.153$, body length $\mathrm{F}=20.07, \mathrm{p}<0.001$, host $\mathrm{F}=3.25, \mathrm{p}=0.041)$. Using the Tukey post hoc test, no statistically significant difference was found between different pairs of $P$. toxostoma, $C$. nasus or hybrids. The same result was found when using endoparasite species richness. However, hybrids tended to harbour fewer endoparasite species and in lower abundance when compared to pure species (Figure 4A).

Because all measures of parasitism, i.e. parasite species richness and parasite abundance, were also affected by locality (ANCOVA, $\mathrm{p}<0.001$ ), we performed the next analysis to eliminate this effect. Manosque, among the localities situated in sympatric zones, was selected because the sample size in this locality was sufficient for statistical evaluation. A significant effect of host on ectoparasite species richness was found (ANCOVA, $F_{4,63}$ total $=9.56, \mathrm{p}<0.001$, heterozygosity $\mathrm{F}=1.45, \mathrm{p}=0.23$, body length $\mathrm{F}=24.52, \mathrm{p}<0.001$, host $\mathrm{F}=3.33, \mathrm{p}=0.042$ ). The Tukey post hoc test revealed a significantly higher ectoparasite species richness in $C$. nasus when compared with $P$. toxostoma $(\mathrm{p}=0.001)$ and hybrids $(\mathrm{p}=0.042)$ (Figure 4B). Unlike $P$. toxostoma and $C$. nasus, their hybrids harbour no endoparasite species. Considering parasite abundance, a significant effect of host on ectoparasite abundance was also found (ANCOVA, $F_{4,63}$ total $=21.10, p<0.001$, heterozygosity $\mathrm{F}=0.68, \mathrm{p}=0.413$, body length $\mathrm{F}=28.06, \mathrm{p}<0.001$, host $\mathrm{F}=17.90$, $\mathrm{p}<0.001$ ). The Tukey post hoc test revealed significantly higher ectoparasite abundance in C. nasus when compared with $P$. toxostoma and hybrids $(\mathrm{p}=0.001)$. The same result was found when using Monogenea (Figure 4C) or Dactylogyrus abundance. Dactylogyrus chondrostomi was not present in hybrids at Manosque.

\section{Discussion}

In this study, we investigated metazoan parasite communities in two intergeneric cyprinid species, native and endemic Parachondrostoma toxostoma and invasive Chondrostoma nasus, sampled in allopatric and sympatric populations. Using mtDNA and microsatellites, we showed that the frequencies of $C$. nasus and $P$. toxostoma in the localities situated in sympatric zones varied from the more abundant $C$. nasus or more abundant $P$. toxostoma to an approximately balanced representation of the two species. This pattern of distribution of these two fish species in the sympatric zone of the Durance River was previously shown by Costedoat et al. [24]. We showed that the frequencies of $C$. nasus and P. toxostoma in the localities studied determine the composition of metazoan parasite communities, i.e. the localities with the highest frequencies of $C$. nasus were the localities with the highest proportion of Monogenea.

Specimens of $P$. toxostoma from the allopatric population were rarely infected by Monogenea. Instead, endoparasite species (i.e. mainly Trematoda) formed the dominant component of parasite communities. By contrast, $C$. nasus specimens from the allopatric population were not parasitized by endoparasite species and, in this case, Monogenea (especially Dactylogyrus species) formed the dominant component of parasite communities. The difference in endoparasite species richness between allopatric $P$. toxostoma and allopatric $C$. nasus could be explained by their different feeding preferences [26-29] (which are linked to different mouth morphology, following Corse et al. [25]) or by different abiotic and biotic factors of their habitats (i.e. the presence of invertebrates like mollusca, isopoda, ostracoda and copepoda, which serve as intermediate hosts for endoparasites). The difference in ectoparasite species richness observed between the allopatric population of P. toxostoma and the allopatric population of $C$. nasus could also indicate that allopatric $P$. toxostoma in southern France is rarely infected by monogenean species or is free of several monogenean species widely infecting $C$. nasus. However, to verify such a hypothesis, the further sampling of $P$. toxostoma from allopatric populations is needed. Our sampling was limited only to one allopatric population, taking into consideration the threatened and protected status of $P$. toxostoma in southern France. Up to now, the investigation of parasite fauna in the allopatric population of $P$. toxostoma has only been performed in the Viaur River (southwest France) by Loot et al. [42], who also found the low prevalence and abundance of Dactylogyrus and Gyrodactylus parasites. However, they found that $P$. toxostoma was infected only by two endoparasite species, which suggests that the endoparasite infection in our allopatric P. toxostoma is more likely the result of environmental conditions of the habitat.

In our study, we showed the high similarity between allopatric and sympatric populations of $C$. nasus, which may suggest that this species expands together with its original parasite fauna. The ectoparasites (especially Dactylogyrus species) maintain a similar intensity of infection in both allopatric and sympatric populations of $C$. nasus; thus, these parasites do not represent a factor limiting the survival and dispersal activity of their host species and their presence is probably the result of co-evolutionary host-parasite 


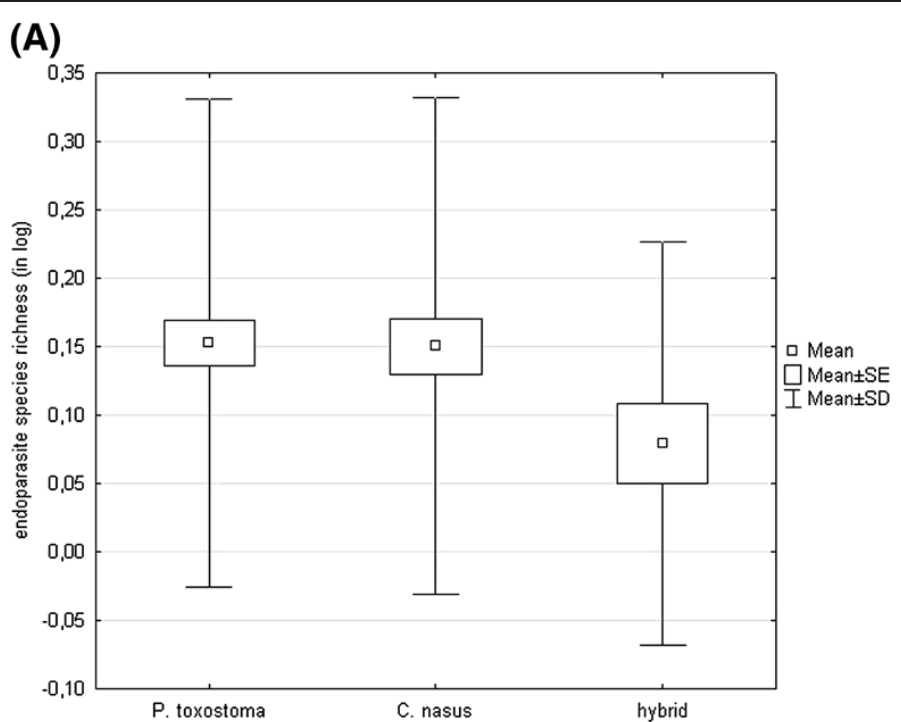

(B)

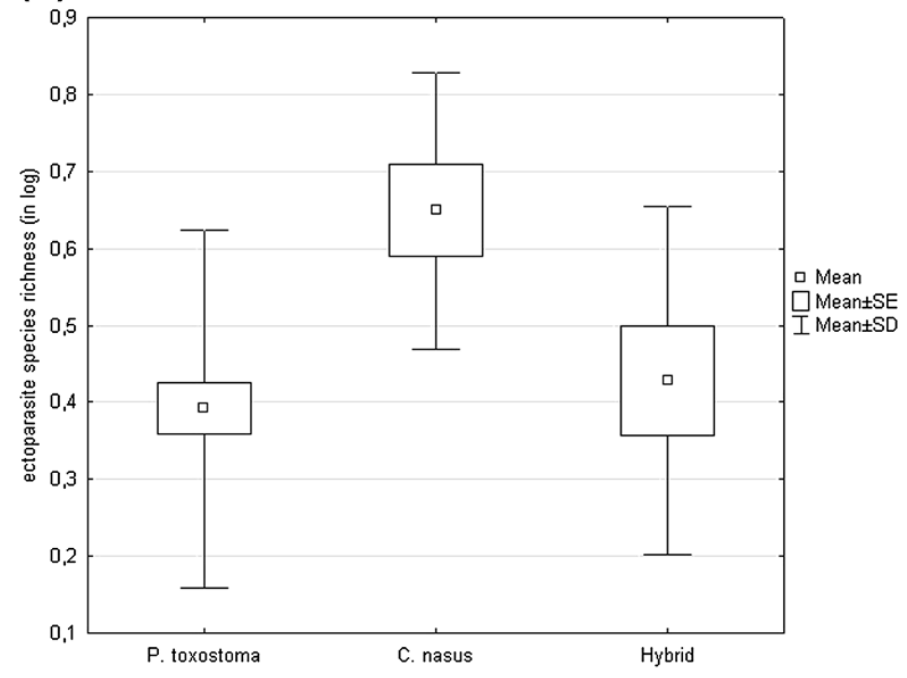

(C)

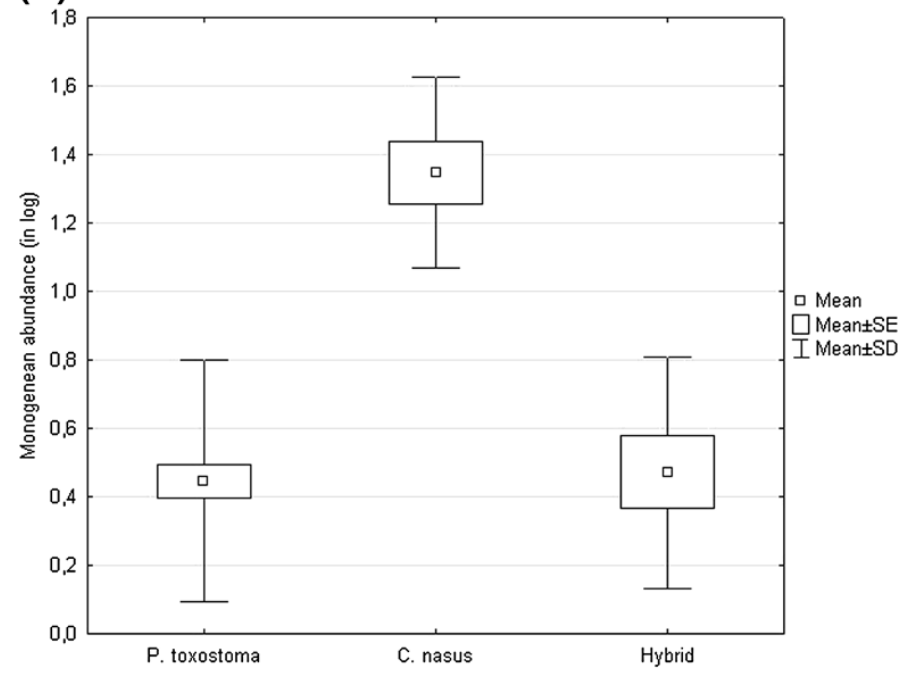

Figure 4 (See legend on next page.) 
(See figure on previous page.)

Figure 4 Short title: Parasitism in P. toxostoma, C. nasus and hybrids. Detail legend: Parasitism in P. toxostoma, C. nasus and hybrids

measured by $(\mathbf{A})$ endoparasite species richness using pooled data from sympatric zones and measured by $(\mathbf{B})$ ectoparasite species richness and (C) monogenean abundance using data from Manosque (a locality in the Durance sympatric zone).

interactions, as shown for Dactylogyrus species and cyprinid fish by Šimková et al. [43]. On the other hand, low similarity in parasite communities between the allopatric and sympatric populations of $P$. toxostoma may indicate that $P$. toxostoma secondarily acquired the parasites (especially Dactylogyrus) after coming into contact with $C$. nasus. From this point of view, the changes in parasite communities in P. toxostoma linked to the invasion of $C$. nasus into areas originally inhabited solely by $P$. toxostoma could represent a danger for native endemic species. However, following the general trend of low Dactylogyrus abundance in P. toxostoma in relation to $C$. nasus observed in sympatric zones, it seems that Dactylogyrus infection probably has a genetic basis limiting the intensity of infection in endemic P. toxostoma. Among Dactylogyrus species, Dactylogyrus chondrostomi was found to be a species infecting $C$. nasus living in allopatric and sympatric populations with a high prevalence. This parasite species was absent in four populations and found only rarely in one population of $P$. toxostoma in sympatric zones, which indicates that $D$. chondrostomi is specific to $C$. nasus. It seems that higher parasite fitness (measured by parasite abundance) in C. nasus is associated with a system of co-adaptation genes.

On the basis of mtDNA and microsatellite data, different genotypes of $P$. toxostoma x C. nasus hybrids were identified. However, our results indicate that the proportion of hybrids and their genotypes across the two sympatric zones (the Durance River and the Ardeche River) are different. These results confirm the findings of a previous study in the Durance hybrid zone [24] and represent the first data obtained from the Ardeche River. The proportions of hybrids were unequal and sometimes low (ranging from 5 to 20\%) depending on the considered localities. This fact together with the absence of $P$. toxostoma or C. nasus in some localities and the low frequencies of one of the Chondrostoma or Parachondrostoma species from other localities in the sympatric zones of the Durance and Ardeche Rivers did not allow us to test simultaneously the effect of locality and host on parasite species richness or abundance. Although parasite load was significantly influenced by the effect of locality, we showed the same pattern of parasite infection in hybrids at two different levels (1) using pooled data and (2) selecting one locality with the largest sample size within the Durance sympatric zone. Thus, $P$. toxostoma $x C$. nasus hybrids were less infected by ectoparasites (and especially by monogeneans of Dactylogyrus genus) than C. nasus. However, as no difference in ectoparasite abundance between hybrids and $P$. toxostoma was found, it seems that Dactylogyrus infection in C. nasus is more likely the result of co-evolutionary history between C. nasus and Dactylogyrus parasites, which limits the infection in both $P$. toxostoma and hybrids. Thus, both $P$. toxostoma and hybrids probably serve as additional hosts for Dactylogyrus. The susceptibility of Salmo salar x Salmo trutta hybrids to Gyrodactylus salaris (highly virulent) and G. derjavini (viviparous Monogenea) was experimentally tested and the intermediate pattern of hybrid susceptibility to that of the parents was shown [44]. They suggested that resistance was transferred through interspecific crosses as a dominant trait. However, our study seems to indicate that the low susceptibility of hybrids to Dactylogyrus infection is linked to the presence of $P$. toxostoma genes in recombinant genotypes. Metazoan parasite communities in another cyprinid hybrid system (Alburnus alburnus $\mathrm{x}$ Rutilus rutilus hybrids from Lake Micri Prespa, Northern Greece), were investigated by Dupont and Crivelli [45]. A higher susceptibility to metazoan parasite infection in hybrids compared to pure species was found for Dactylogyrus and Diplozoon species (Monogenea), Bolbophorus confusus (larval stages of Trematoda) and Pomphorhynchus bosniacus (Acanthocephala). This was explained by the spatial and trophic positions of the hybrids, which were intermediate between the two pure species. Concerning P. toxostoma $\times$ C. nasus, Corse et al. [30] concluded that pure and hybrid specimens in the hybrid zone exhibit more diverse feeding behaviour than in the allopatric zone consistent with generalist behaviour. $P$. toxostoma $\mathrm{x} C$. nasus hybrids are not intermediate between pure species and hybrids have "super $P$. toxostoma" feeding behaviour i.e. they feed on fewer diatomes and more invertebrates than both pure species [46]. However, in our study, there was a trend towards lower endoparasite abundance in hybrids compared to both P. toxostoma and C. nasus, suggesting that there is no link between the frequencies of invertebrates (as the potential intermediate hosts) in the food and infection by endoparasite species in sympatric zones.

Concerning Monogenea, the strictly host-specific Dactylogyrus in the study by Dupont and Crivelli [44] achieved lower prevalence in Alburnus alburnus $\mathrm{x}$ Rutilus rutilus hybrids compared to pure species. A similar result, i.e. the effect of introgression rate on 
Diplozoon infection (Monogenea) in the hybrids of Barbus barbus and B. meridionalis from a hybrid zone in southern France, was found, in which parasite prevalence correlated with the percentage of $B$. meridionalis genes [47]. In our study, Dactylogyrus chondrostomi, a parasite specific to C. nasus, was absent in hybrids reported in five localities where both host fish species live in sympatry, and its presence was confirmed only in hybrids at Avignon, a single locality investigated in the sympatric zone on the Durance River, where $92 \%$ of collected specimens were determined as C. nasus. The high frequency of $C$. nasus seems to represent a more plausible explanation for the presence of $D$. chondrostomi in hybrids.

\section{Conclusions}

We can conclude that the compositions of parasite communities in allopatric populations of $C$. nasus and $P$. toxostoma are very different, probably because of different feeding preferences or different abiotic and biotic characters of habitat (like the presence of intermediate hosts for endoparasites). However, they are also related to hostparasite co-evolutionary history, when the presence of a specific Dactylogyrus species on C. nasus seems to be the result of co-adaptation interactions. Our results suggest that C. nasus is a source of infection of Dactylogyrus parasites and has an impact on native and protected $P$. toxostoma with respect to their transmission. The genotype of $P$. toxostoma and recombinant genotypes of hybrids, even if they are susceptible to Dactylogyrus, have lower levels of Dactylogyrus infection than C. nasus.

\section{Competing interests}

The authors declare that they have no competing interests.

\section{Authors' contributions}

AS designed this study and drafted the manuscript. AŠ, MD, MO, RC, CC and AG participated on the field studies to acquire fish and parasite data. PN with the help of MD and MO determined the parasites. MS and CC carried out the molecular analyses and statistics on molecular data. AS analyzed the whole data. MD, MO, CC and AG have been involved in drafting the manuscript or revising it critically for important intellectual content. All authors read and approved the final version of manuscript.

\section{Acknowledgements}

This study was funded by the Czech Science Foundation, Project No. P505/ 10/1138. We thank Milan Gelnar, Jarek Piňos, Radim Blažek, Eva Řehulková, Nad’a Musilová, Kateřina Francová, Kristýna Hejlová and Lucie Havlátová for their help with fish dissection and parasite collection. Warm thanks go to JP Cornec for help in field assistance. We are very grateful to Matthew Nicholls for the English revision of the draft.

\section{Author details}

'Department of Botany and Zoology, Faculty of Science, Masaryk University, Kotlářská 2, 61137 Brno, Czech Republic. ${ }^{2}$ Aix-Marseille Université, IMBE, UMR CNRS 7263, Evolution Génome Environnement, Case 36, 3 Place Victor Hugo, 13331 Marseille Cedex 3, France.

Received: 25 May 2012 Accepted: 24 August 2012 Published: 11 September 2012

\section{References}

1. Bohn T, Amundsen PA: The competitive edge of an invading specialist. Ecology 2001, 82:2150-2163.

2. Kelly DW, Patterson RA, Townsend CR, Poulin R, Tompkins DM: Parasite spillback: A neglected concept in invasion ecology? Ecology 2009, 90:2047-2056.

3. Dunn AM: Parasites and biological invasions. Adv Parasit 2009, 68:161-184.

4. Poulin R, Paterson RA, Townsend CR, Tompkins DM, Kelly DW: Biological invasions and the dynamics of the endemic diseases in freshwater ecosystems. Freshwater Biol 2011, 56:676-688.

5. Keane RM, Crawley MJ: Exotic plants invasions and the enemy release hypothesis. Trends Ecol Evol 2002, 17:164-170.

6. Torchin ME, Lafferty KD, Dobson AP, McKenzie VJ, Kuris AM: Introduced species and their missing parasites. Nature 2003, 421:628-630.

7. Colautti Rl, Ricciardi A, Grigorovich IA, Madlsaac HJ: Is invasion success explained by the enemy release hypothesis? Ecol Lett 2004, 7:721-733.

8. Prenter J, MacNeil C, Dick JTA, Dunn AM: Roles of parasites in animal invasions. Trends Ecol Evol 2004, 19:385-390.

9. Taraschewski H: Host and parasites as aliens. J Helminthol 2006, 80:99-128.

10. Elvira B, Almodovar A: Freshwater fish introductions in Spain: facts and figures at the beginning of the $21^{\text {st }}$ century. J Fish Biol 2001, 59:232-331.

11. Moulia C, Le Brun N, Loubes C, Marin R, Renaud F: Hybrid vigor against parasites in interspecific crosses between 2 mice species. Heridity 1995, 74:48-52.

12. Goldberg SR, Bursey CR, Malmos KB, Sullivan BK, Cheam H: Helminths of the southwestern toad, Bufo microscaphus, woodhouse's toad, Bufo woodhousii (Bufonidae), and their hybrids from central Arizona. Great Basin Nat 1996, 56:369-374

13. Hedrick RP, Waltzek TB, McDowell TS: Susceptibility of koi carp, common carp, goldfish, and goldfish $\mathrm{x}$ common carp hybrids to cyprinid herpesvirus-2 and herpesvirus-3. J Aquat Anim Health 2006, 18:26-34.

14. Sage RD, Heynemen D, Lim KC, Wilson AC: Wormy mice in hybrid zone. Nature 1986, 324:60-63.

15. Moulia C, Aussel JP, Bonhomme F, Boursot P, Nielsen JT, Renaud F: Wormy mice in a hybrid zone: a genetic control of susceptibility to parasite infection. J Evol Biol 1991, 4:679-687.

16. Moulia C, Le Brun N, Dallas J, Orth A, Renaud F: Experimental evidence of genetic determinism in high susceptibility to intestinal pinworms infection in mice: a hybrid zone model. Parasitology 1993, 106:387-393.

17. Derothe J-M, Loubes C, Perriat-Sanguinet M, Orth A, Moulia C: Experimental trypanosomiasis of natural hybrids between House Mouse subspecies. Int J Parasitol 1999, 29:1011-1016.

18. Derothe J-M, Le Brun N, Loubes C, Perriat-Sanguinet M, Moulia C: Susceptibility of natural hybrids between house mouse subspecies to Sarcocystis muris. Int J Parasitol 2001, 31:15-19.

19. Matondo BN, Ovidio M, Poncin P, Kakesa TA, Wamuini LS, Philippart JC Hybridization success of three common European cyprinid species, Rutilus rutilus, Blicca bjoerkna and Abramis brama and larval resistance to stress tests. Fisheries Sci 2007, 73:1137-1146.

20. Vetešník L, Halačka K, Papoušek I, Mendel J, Šimková A: The first record of a natural hybrid of the roach Rutilus rutilus and nase Chondrostoma nasus in the Danube River Basin, Czech Republic: morphological, karyological and molecular characteristics. J Fish Biol 2009, 74:1669-1676.

21. Toscano BJ, Pulcini D, Hayden B, Russo T, Kelly-Quinn M, Mariani S: An ecomorphological framework for the coexistence of two cyprinid fish and their hybrids in a novel environment. Biol J Linn Soc 2010, 99:768-783.

22. Crespin L, Berrebi P, Lebreton J-D: Asymmetrical introgression in a freshwater fish hybrid zone as revealed by a morphological index of hybridization. Biol J Linn Soc 1999, 67:57-72.

23. Costedoat C, Pech N, Chappaz R, Salducci MD, Lim P, Gilles A: Étude de I' hybridation introgressive entre Chondrostoma t. toxostoma et Chondrostoma n. nasus (Téléostéen, Cyprinidae) en utilisant une approach multiple. Cybium 2004, 28:51-61.

24. Costedoat C, Pech N, Chappaz R, Gilles A: Novelties in hybrid zones: crossroads between population genomic and ecological approaches. PLoS One 2007, 2(4):e357.

25. Corse E, Nève G, Sinama M, Pech N, Costedoat C, Chappaz R, Gilles A: Plasticity of ontogenetic trajectories in cyprinids: a source of 
evolutionary novelties. Biol J Lin Soc 2012, 106:342-355.

26. Chappaz R, Brun G, Olivari G: New data about biology and ecology of a little-studied fish: Parachondrostoma toxostoma (Vallot, 1836). Comparison with nase, Chondrostoma nasus (L.,1766). Comp Rend Acad Sci, Ser 3 1989, 309:181-186.

27. Corse E, Costedoat C, Chappaz R, Pech N, Martin JF, Gilles A: A PCR-based method for diet analysis in freshwater organisms using 18SrDNA barcoding on faeces. Mol Ecol Res 2010, 10:96-108.

28. Nelva A: Biogéographie, Démographie et Écologie de Chondrostoma nasus (L., 1758) (Hotu, Poisson, Teléosteen, Cyprinidé). Lyon: PhD thesis, Université Claude Bernard - Lyon I; 1985. in French.

29. Freyhoff J: Feeding traces as an indicator of habitat choice in nase, Chondrostoma nasus. Folia Zool 1995, 44:57-61.

30. Corse E, Costedoat C, Pech N, Chappaz R, Grey J, Gilles A: Trade-off between morphological convergence and opportunistic diet behaviour in fish hybrid zone. Front Zool 2009, 6:26.

31. Ergens R, Lom J: Causative agents of fish diseases. Prague: Academia; 1970 (in Czech).

32. Rohlenová K, Morand S, Hyršl P, Tolárová S, Flajšhans M, Šimková A: Are fish immune systems really affected by parasites? An immunoecological study of common carp (Cyprinus carpio). Parasites and Vectors 2011, 4:120

33. Costedoat C, Pech N, Salducci MD, Chappaz R, Gilles A: Evolution of mosaic hybrid zone between invasive and endemic species of Cyprinidae through space and time. Biol J Linn Soc 2005, 85:135-155.

34. Dubut V, Sinama M, Martin JF, Meglecz E, Fernandez J, Chappaz R, Gilles A, Costedoat C: Cross-species amplification of 41 microsatellites in European cyprinids: A tool for evolutionary, population genetics and hybridization studies. BMC Res Notes 2010, 3:135.

35. Chatterji S, Pachter L: Reference based annotation with GeneMapper. Genome Biol 2006, 7:R29.

36. Van Oosterhout C, Hutchinson WF, Wills DPM, Shipley P: MICRO-CHECKER: software for identifying and correcting genotyping errors in microsatellite data. Mol Ecol Notes 2004, 4:535-538.

37. Anderson EC, Thompson EA: A model-based method for identifying species hybrids using multilocus genetic data. Genetics 2002, 160:1217.

38. Szpiech ZA, Jakobsson M, Rosenberg NA: ADZE: a rarefaction approach for counting alleles private to combinations of populations. Bioinformatics 2008, 24:2498

39. Excoffier L, Laval G, Schneider S: Arlequin (version 3.0): an integrated software package for population genetics data analysis. Evol Bioinform 2005, 1:47.

40. Coulon A: GENHET: an easy-to-use $R$ function to estimate individual heterozygosity. Mol Ecol Res 2010, 10:167-169.

41. Magurran A: Ecological diversity and its measurement. London: Croom. Helm; 1988

42. Loot G, Reyjol Y, Poulet N, Šimková A, Blanchet S, Lek S: Effects on small weirs on fish parasite communities. Parasitol Res 2007, 101:775-489.

43. Šimková A, Morand S, Jobet E, Gelnar M, Verneau O: Molecular phylogeny of congeneric monogenean parasites (Dactylogyrus): a case of intra-host speciation. Evolution 2004, 58:1001-1018.

44. Bakke TA, Soleng A, Hartus PD: The susceptibility of Atlantic salmon (Salmo salar L.) x brown trout (Salmo trutta L.) hybrids to Gyrodactylus salaris Malmberg and Gyrodactylus derjavini Mikailov. Parasitology 1999, 119:467-481.

45. Dupont $F$, Crivelli AJ: Do parasite confer a disadvantage to hybrids? A case study of Alburnus alburnus $\times$ Rutilus rubilio, a natural hybrid of Lake Mikcri Prespa, Northern Greece. Oecologia 1988, 75:587-592.

46. Corse E: Le "barcoding alimentaire": développement d'un nouvel outil d'écologie moléculaire pour les milieux d'eau douce. Exemple au travers de létude de la zone hybride Durance entre les deux Chondrostomes français. Marseille: PhD thesis. Université de Provence; 2010. in French.

47. Le Brun N, Renaud F, Berrebi P, Lambert A: Hybrid zones and host-parasite relationships: effect on the evolution of parasitic specificity. Evolution 1992, 46:56-61.

\section{doi:10.1186/1756-3305-5-200}

Cite this article as: Šimková et al.: Does invasive Chondrostoma nasus shift the parasite community structure of endemic Parachondrostoma toxostoma in sympatric zones?. Parasites \& Vectors 2012 5:200.

\section{Submit your next manuscript to BioMed Central and take full advantage of:}

- Convenient online submission

- Thorough peer review

- No space constraints or color figure charges

- Immediate publication on acceptance

- Inclusion in PubMed, CAS, Scopus and Google Scholar

- Research which is freely available for redistribution 